\title{
Improving the health and quality of life of cancer survivors through exercise: The role of the primary care physician
}

\author{
James R. Vallerand
}

\section{University of Alberta}

An estimated 187,600 new cases of cancer were diagnosed in Canada in the year 2013 alone. ${ }^{1}$ Treatment options for cancer patients are continually improving, leading to greater survivorship amongst numerous different cancer groups. As a result, an increased demand is being placed on primary care physicians to help manage these patients' quality of life by treating numerous cancer-related comorbidities associated with life beyond cancer-specific diseases. Many cancer survivor groups experience debilitating levels of fatigue; for example, up to $90 \%$ of lung cancer survivors experience fatigue that persists for months posttreatment. ${ }^{2}$ These cancer-related fatigue levels are often linked to a reduction in exercise capacity, which places these particularly vulnerable individuals at a heightened risk for developing conditions such as cardiovascular disease and hypertension, and can ultimately contribute to an overall poor quality of life or even premature death. ${ }^{3}$

One key area in which primary care physicians may be able to attenuate or reverse the effects of these cancerrelated comorbidities is through the prescription of physical activity. The consensus amongst experts remains that physical activity is safe for cancer patients across the entire cancer continuum, ${ }^{4}$ and cancer survivors who meet the recommended levels of physical activity report significantly less fatigue, better overall physical health, and an improved short and long term quality of life. ${ }^{2,3}$ Though physical activity may serve as viable conduit for improving cancer survivors' overall health, ${ }^{5}$ the most effective ways to initiate and maintain health-promoting physical activity levels for these survivors remains largely unknown. Researchers contend that physical activity interventions are likely to yield the greatest results and applicability when they are theory driven and targeted to meet the specific psychosocial needs of cancer survivor populations. ${ }^{2}$ However, inconclusive results stemming from theoreticallydriven interventions may explain why physician counseling for physical activity remains scarce despite the pivotal role primary health care providers may have in increasing their patients' physical activity behaviour.

Theoretically-driven efforts to improve cancer survivors' dwindling physical activity rates in hopes of promoting greater overall health typically focus on strategies to increase individuals' intentions to be active. Though physical activity intentions remain as one of the strongest correlates of physical activity participation, ${ }^{6}$ it appears that when moderately sized changes to intentions are made through interventions, only trivial-sized changes in physical activity participation. ${ }^{7}$ Furthermore, in most intention-focused studies, a near-even split exists amongst highly intentional individuals, where half become physically active, and the other half abstain from physical activity participation. ${ }^{6}$ Thus, other physical activity promoting variables seem to be at play, and yet remain largely under studied. While additional research is being conducted to discover and validate the best ways to get cancer survivors more active for the long-term, current efforts should seek to apply simplified strategies in order to produce immediate healthenhancing effects.

Notwithstanding the current dearth of research focusing on this problem space, the primary health care physician may still have a role in applying strategies that can promote healthenhancing levels of physical activity to their cancer survivor patients. For example, when an oncologist recommends to their patients to exercise everyday throughout the week, significant and meaningful improvements in their patients' exercise levels are noted. ${ }^{8}$ However, since primary care physicians act as the first point of contact and likely meet with their patients more often than would an oncologist, their ability to expose their patients to expert advice may even be potentially superior. Thus, it seems possible that if primary health care physicians simply recommend that their patients become more physically active, these 
cancer survivors may become motivated enough to not only want to be more active but moreover to actually engage in the recommended physical activity.

Despite depicting the influential status that primary care physicians may hold in promoting health-facilitative physical activity in their cancer survivor patients, some physicians may remain reluctant to provide specific physical activity recommendations to their patients. Therefore, the necessary tools and resources need to continually be made available for uptake by primary health care services, and must remain clearly interpretable for practitioners. In sum, if primary healthcare physicians recommend that their patients increase their daily levels of physical activity, numerous health benefits can arise and ultimately result in a higher quality of life post-treatment. Thus, considering that cancer survivors' quality of life is likely to diminish if they remain sedentary, primary health care physicians should feel empowered in recommending that their patients become more physically active.

\section{Acknowledgements}

Special thanks to Dr. Kerry S. Courneya for his thoughtful guidance and insight on the topic presented in this article.

\section{References}

1. Canadian Cancer Society's Advisory Committee on Cancer Statistics. Canadian cancer statistics 2013. Toronto: Canadian Cancer Society; 2013. [cited 2014 Jan 28]. Available from: http://www.cancer.ca/ /media/cancer. $\mathrm{ca} / \mathrm{CW} /$ cancer\%20information/cancer\%20101/Canadian\%20cancer\%20 statistics/canadian-cancer-statistics-2013-EN.pdf

2. Coups EJ, Park BJ, Feinstein MB, Steingart RM, Egelston BL, Wilson DJ, Ostroff JS. Correlates of physical activity among lung cancer survivors. Psycho-Oncology. 2009; 18: 395-404.

3. Mishra SI, Scherer RW, Geigle PM, Berlanstein DR, Topaloglu O, Gotay $\mathrm{CC}$, et al. Exercise interventions on health-related quality of life for cancer survivors. Cochrane Database of Systematic Reviews 2012, Issue 8. Art. No.: CD007566. DOI: 10.1002/14651858.CD007566.pub2.

4. Peddle-McIntyre CJ, Bell G, Fenton D, McCargar L, Courneya KS. Changes in motivational outcomes after a supervised resistance exercise training intervention in lung cancer survivors. Cancer Nurs. 2012; 00: 1-9.

5. Speed-Andrews AW, Courneya KS. Effects of exercise on quality of life prognosis in cancer survivors. Curr Sports Med Rep. 2009; 8: 176-181.

6. Rhodes RE, de Bruijn G. How big is the physical activity intention-behaviour gap? A meta-analysis using the action control framework. Br J Health Psychol. 2013; 18: 296-309.

7. Rhodes RE, Dickau L. Experimental evidence for the intention-behavior relationship in the physical activity domain: a meta-analysis. Health Psychol. 2012: 31: 724-727.

8. Jones LW, Courneya KC, Fairey AS, Mackey JR. Does the theory of planned behavior mediate the effects of an oncologist's recommendation to exercise in newly diagnosed breast cancer survivors? Results from a randomized controlled trial. Health Psychol. 2005; 24(2): 189-197.

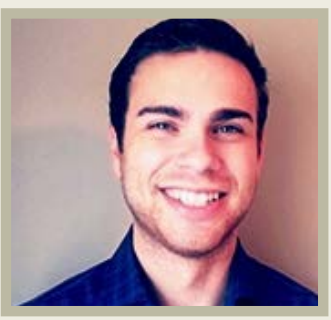

\section{James R. Vallerand}

James Vallerand is a PhD student studying Behavioural Medicine in the Faculty of Physical Education and Recreation at the University of Alberta. James' research focuses on cancer survivorship and exercise oncology, with a specific concentration on exploring what motivates cancer survivors to adhere to exercise programs and how this may relate to improving health outcomes. 